\title{
Streptococcus viridans
}

National Cancer Institute

\section{Source}

National Cancer Institute. Streptococcus viridans. NCI Thesaurus. Code C124413.

A non-taxonomic grouping of Streptococcal bacteria that are either alpha-hemolytic, producing a green coloration on blood agar plates, or nonhemolytic. Within this heterogeneous group, some species are commensal flora while others are human pathogens. 